\title{
Strategies for Implementation of Innovation by Wine Producers in Bulgaria: An Empirical Evidence
}

\author{
Mina Angelova ${ }^{1 *}$ and Daniela Pastarmadzhieva ${ }^{2}$ \\ ${ }^{1}$ University of Plovdiv Paisii Hilendarski, Department of Management and Quantitative Methods in \\ Economics, 24 Tsar Asen Str., Plovdiv 4000, Bulgaria \\ ${ }^{2}$ University of Plovdiv Paisii Hilendarski, Department of Political Sciences and National Security, 24 \\ Tsar Asen Str., Plovdiv 4000, Bulgaria
}

\begin{abstract}
The innovation strategy is essential for the effective implementation of innovations for various reasons. Thus, the aim of the current study is to identify the level of implementation of innovation strategies among Bulgarian wine producers, and to identify an association between various factors and the intention to implement innovation strategies. The object of the research is Bulgarian SMEs wine producers. The focus is their activity, regarding the innovation strategy of their company. The results show that several associations can be identified as regards some of the characteristics of the SMEs.
\end{abstract}

\section{Introduction}

The innovations are rather important for the competitiveness of a company. As wine production is a traditional economic sector in Bulgaria its sustainable development and competitiveness can be enhanced by implementation of innovations. However, the latter should be a consistent and controlled process. Making the implementation of innovation resultative and successful depends on the existence of an innovation strategy.

Thus, the aim of the current study is to identify if Bulgarian wine producers who are SMEs, implement an innovation strategy and if there are factors which associate with the implementation of strategies and readiness to implement such. The object of the study is Bulgarian SMEs wine producers. The focus is their activity regarding the innovation strategy of their company. The results are obtained through online survey performed in the period December 2019 - January 2020 which present a structured interviews using own questionnaire. The collected data is processed with IBM SPSS Statistics and frequencies and cross tables are used.

The article is structured as follows: following the introduction in the second part is described a conceptual framework of innovation strategies. The latter paves the way for the third part in which is presented the methodology of the study. The fourth part introduces the obtained results of the survey and discussion based upon the figures. The paper closes with

* Corresponding author: mina.angelova@uni-plovdiv.bg 
conclusions and recommendations for future research in the field of investigation the innovation strategies in wine industry.

\section{Innovation Strategy - a Conceptual Framework}

The innovations are object of number of studies and their relevance for the competitiveness of a company is inevitable [1]. Recent years the number of studies which investigate the importance of implementing an innovation strategy increases. In fact, when we speak of innovation strategy this may include national [2], sub-national [3], supranational [4] or of a company [5]. The focus of the current study are the essentials and the importance of the innovation strategy for a company. Furthermore, here we distinguish between companies, who create innovations [6], especially concerning the information and communication technologies, and those who implement innovations developed by another company [7]. Thus, in the first case it is strategy for developing innovation and in the second - a strategy for implementation of innovations. In the current study we use the term "innovation strategy", regarding the latter meaning.

While defining the notion of innovation strategy Sauber and Tschirky show why it is important. According to them it represents a kind of guide, which sets the course of development, thus allowing the efforts to be focused. Alongside, it contributes to building the image of a company and ensures sustainability in the implementation of innovations. Furthermore, the innovation strategy considers "integral innovations, innovation barriers, and the degree of newness of the innovation as well as the required innovation relevant knowledge." [8]

Furthermore, Vanhaverbeke and Peeters also stress on the role of the strategy for giving a direction of companies' innovation activity. However, they also draw the attention to the need of flexibility to enhance their current activity to cover the future development strategy of the company. [5]

Stankevice and Jucevicius performed a research to create a conceptual framework for the innovation strategy. They made a review of various works on the topic and suggested a conceptual model with several elements. According to them an innovation strategy should consist of strategy's objectives, arenas/objects, vehicles/resources, differentiators, speed/scope and general logic. [9]

Thus, the most important elements of strategy for implementing innovations should be aims, means and schedule. Alongside, it should be adapted to new realities as such occur. However, the studies focused on the implementation of innovations identify one significant factor, which should be considered by the companies, when they prepare their innovation strategies.

Saleh and Wang warn that the strategy "does not operate in a vacuum". The authors are talking about the role of the context in the company. According to them the strategy should be adapted to the specific environment in the company, the values, and practices of its staff. The strategy and the environment should coincide to ensure the efficiency of the implementation of the strategy. [10]

The specific organizational climate is a prerequisite not only for the companies, who develop innovations, but also for those who intend to implement such.

Belasen and Luber shared similar ideas. They believe that the middle managers have a crucial role in the successful adoption of new ideas as part of the strategy for implementing innovations. They can help these new ideas to be adapted and presented properly to the people working in the company in order the ideas to be accepted. The authors stress on the importance of the organization culture of the company and need for compliance between the strategy and the culture. [11] 
In another studies similar results are acquired. Kim and Chung identify individual factors as rather significant for the success of implementing innovations in a company. [12]

\section{Methodology of the Study}

The results presented in the current paper are part of a project. The latter on one hand aims to establish the extent to which innovations in the wine sector are applied and on the other to offer opportunities for increasing the innovation activity of the enterprises from the examined sector. The methodology of the study includes three stages. The first one is survey and the second in-depth interviews. Their methodology is described in detail in our paper "Conceptual Model for Investigating the Innovation Activity of Enterprises" [13]. Furthermore, the third stage consists of interviews with experts performed after the collection of data from the in-depth interviews. The experts are scholars and experts in the area of innovation, competitiveness, and statistics. Based on surveys and in-depth interviews questions and the collected data, they provide opinions, ideas and new directions as regards the processing of the data.

In the current paper are presented some of the results of the survey performed in the period December 2019 - January 2020. In the latter all the SMEs wine producers were invited to take part in the online survey by e-mail and some of them were personally contacted. As a result, 156 online questionnaires were sent by e-mail and 57 of them participated in the survey which represents $37 \%$ response rate. Thus, the collected data provides relevant results. The statistical methods used in the study are frequencies and cross tables. The latter were used to identify if there is an association between the existence of innovation strategy in the company plus the readiness to implement such and various factors. The examined factors are years of functioning of the enterprise, size of enterprise, additional activities, markets for production realization, sources of financing, sources of raw material, and types of produced wine.

\section{Results and Discussion}

As mentioned above, the innovation strategies are the basis of the innovation activity. Thus, we aimed at identifying if there is an association between number of factors and the implementation of innovation strategies.

The results on Fig. 1 show that most of the wine producers (58\%) already have innovation strategy in their company which is rather positive indicator. Furthermore, when we add those who plan to do it, it is obvious that as much as $3 / 4$ of the studied wine producers implement or plan to implement innovation strategy. This is the group of wine producers, who we decided to refer to as strategic innovators. Based upon an official definition, a Strategic Innovation is a future-focused business development framework that identifies breakthrough growth opportunities, accelerates business decisions, and creates near-term, measurable impact within the context of a longer-term vision for sustainable competitive advantage. [14] The obtained results confirm that wine producers can be referenced as mentioned above. 


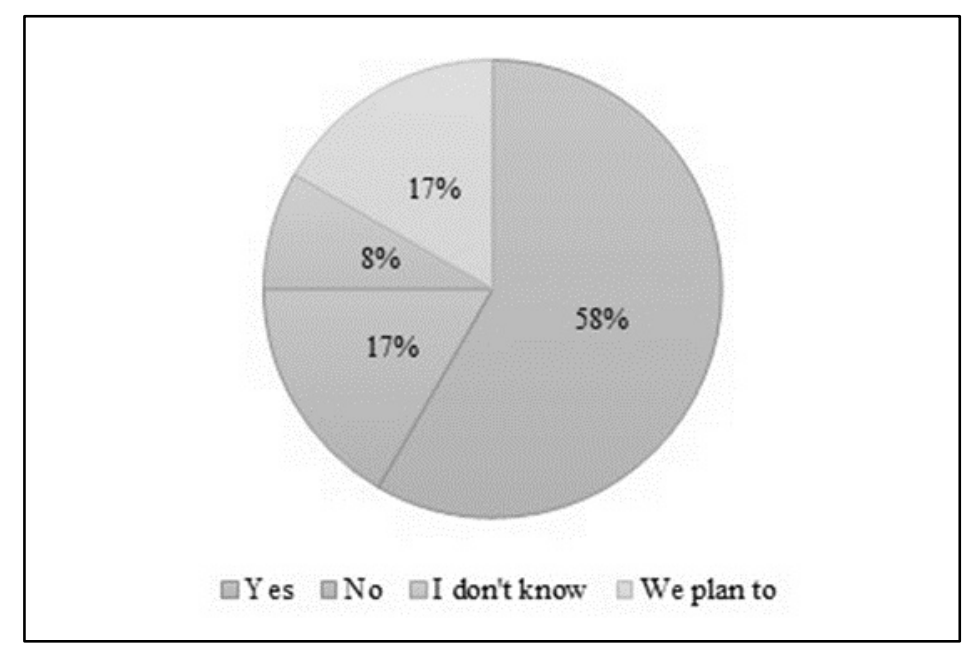

Fig. 1. Implementation of innovation strategies by wine producers Source: Own data

The results show that such strategic innovators can be found mostly in the newest companies, which are between 6 and 10 years old (Fig. 2). The share of those who have or intend to have innovation strategy in this group of companies is $86 \%$. It decreases in the companies with more years of functioning and in those with more than 15 years of experience their share is $55 \%$.

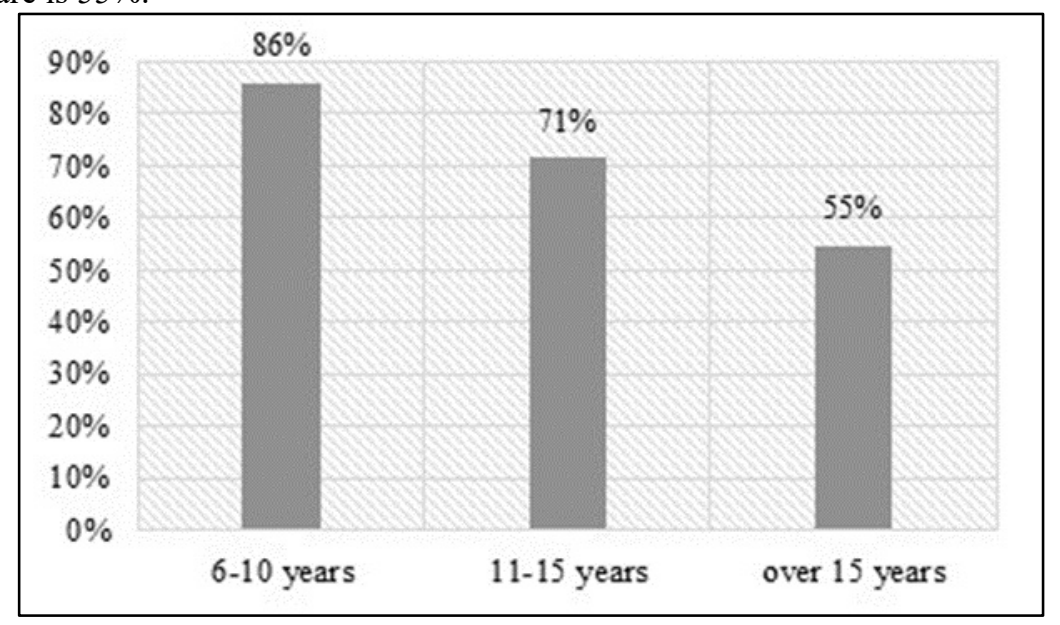

Fig. 2. Years of functioning of the enterprise and implementation of innovation strategies Source: Own data

Similar dependency is also identified as concerns the size of the enterprises. The smallest wine producers, with up to 10 people in their company have the largest share of strategic innovators. They are $72 \%$ and the smallest share is in the enterprises, sized between 11 and 49 people. (Fig. 3) 


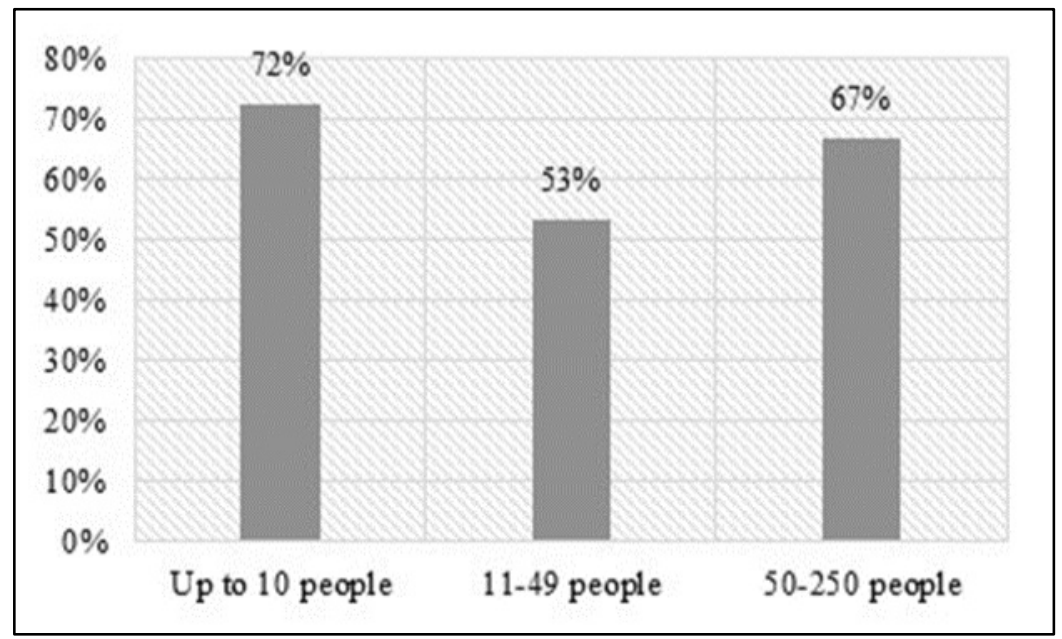

Fig. 3. Size of the enterprise and implementation of innovation strategies Source: Own data

As we wanted to identify if the additional activities are related to the share of strategic innovators, we asked the respondents what other activities do they have in addition to the wine production. Fig. 4 shows that they are distributed quite evenly across the groups. The highest rate is among those who do wine tourism $(68 \%)$ and the lowest is among those who grow vines $(64 \%)$.

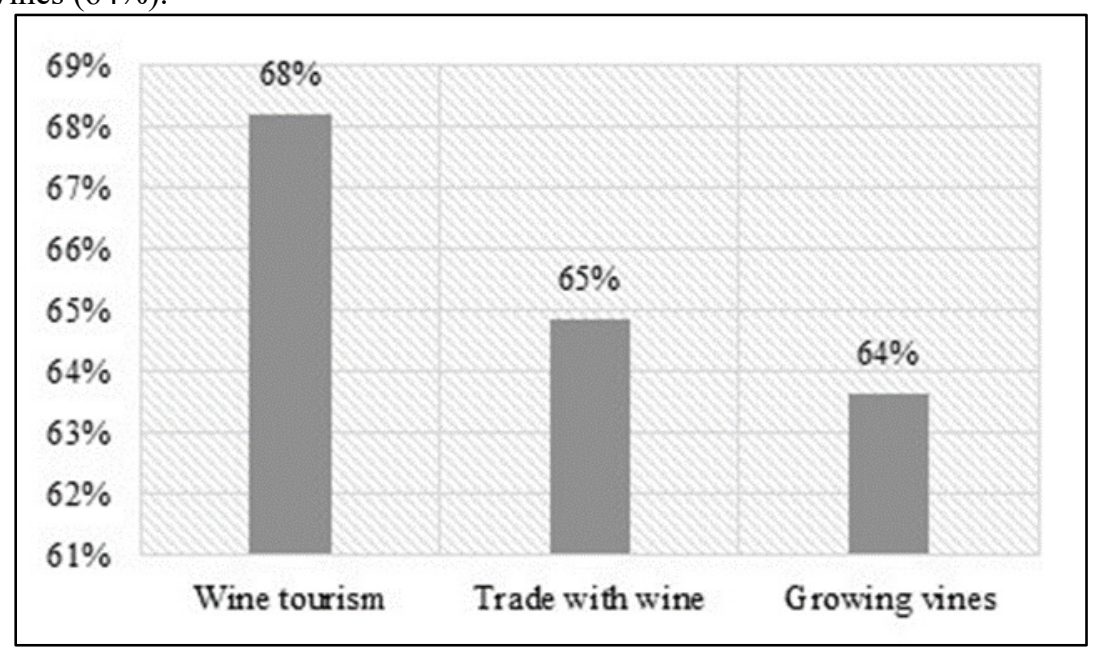

Fig. 4. Additional activities and implementation of innovation strategies Source: Own data

Fig. 5 indicates that the strategic innovators have the highest representation among the companies who sell their production worldwide (78\%). Among those, who sell in European and Bulgarian markets, their share is close, respectively $68 \%$ and $66 \%$. 


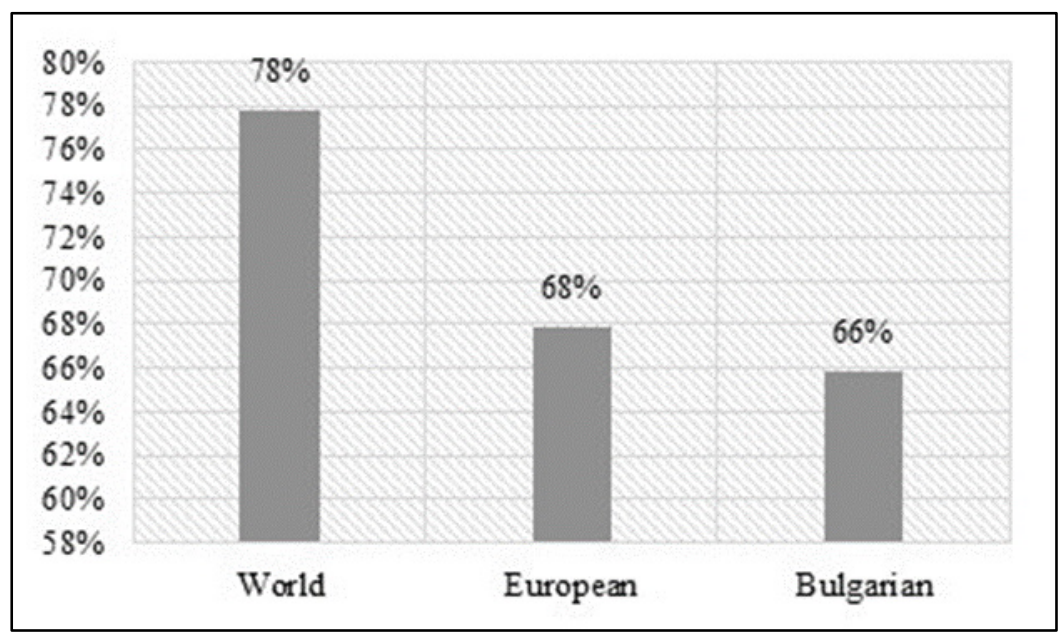

Fig. 5. Markets for product realization and implementation of innovation strategies Source: Own data

To some extent there is an association between the sources of financing and the implementation or readiness for implementation of innovation strategy. The strategic innovators are most among the wine producers whose source of financing are national programs. The group of those who use their own funds (68\%) follows them. There are $65 \%$ strategic innovators among the wine producers, who use bank credits. Their share is lowest among those, who use European Funds (64\%). (Fig. 6)

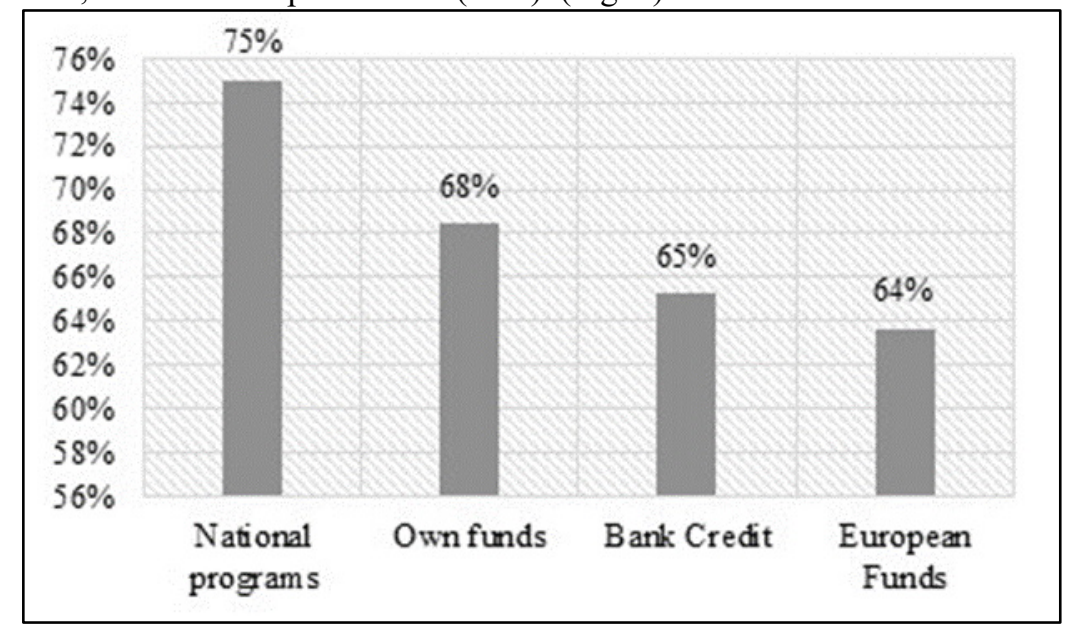

Fig. 6. Sources of financing and implementation of innovation strategies Source: Own data

The results displayed on Fig. 7 illustrate an interesting association. $73 \%$ of those who use raw material (grape) from Bulgarian suppliers and $70 \%$ of those who have their own vineyards are strategic innovators. Their share in these groups is significantly higher than the share among the wine producers who use imported raw material (50\%). 


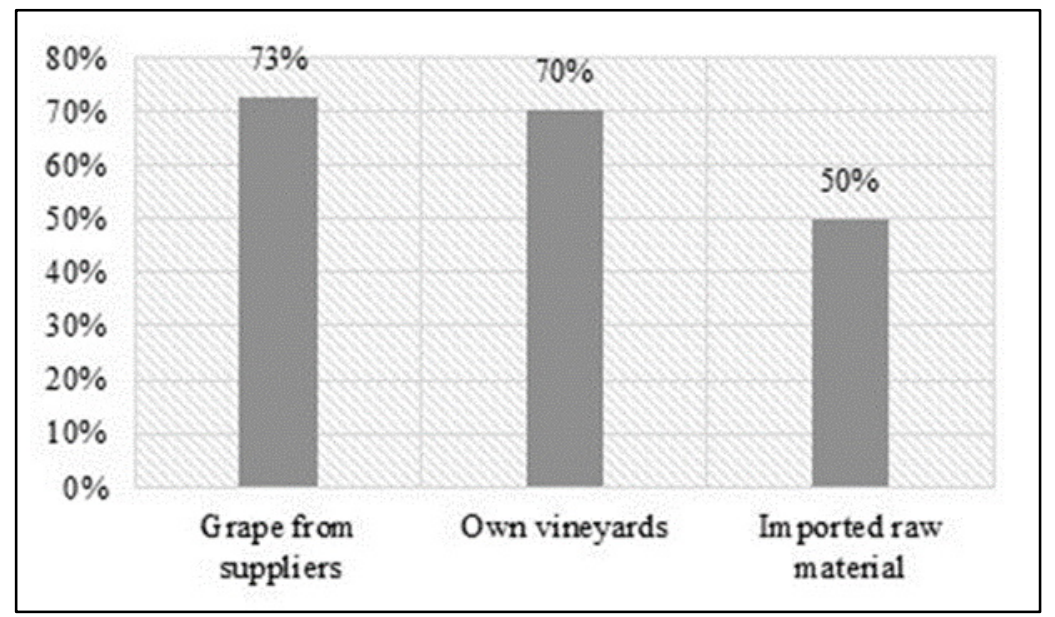

Fig. 7. Sources of raw material and implementation of innovation strategies Source: Own data

Fig. 8 shows how the type of produced wine is associated with the implementation and readiness to implement innovation strategies. There are certain types of produced wine for which all of the respondents who produce them are strategic innovators $(100 \%)$. These are the producers of fruit wines, special wines and wines with PDO. Sparkling wines and organic (bio) wines are produced respectively by $86 \%$ and $80 \%$ strategic innovators. The lowest share of strategic innovators can be found among those, who produce still wines.

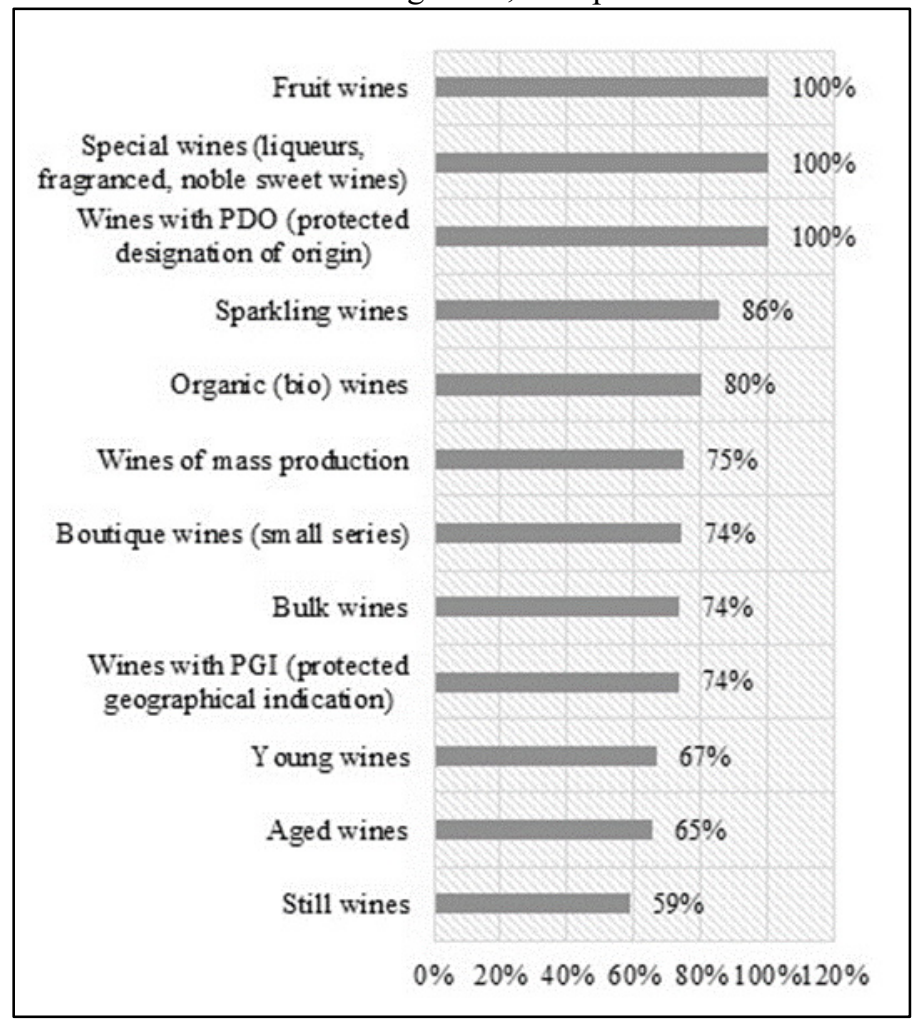

Fig. 8. Types of produced wines and implementation of innovation strategies 
Source: Own data

\section{Conclusion}

The innovations are crucial for the competitiveness of a company and the innovation strategy can make the implementation of innovations successful. The most important requisites of the strategy are its goals, means and schedule. Furthermore, the innovation strategy should be in line with the culture of the company to be accepted and implemented by the employee.

Bulgarian wine producers tend to implement innovations and as some preliminary results showed these are mostly marketing and product innovations [15]. However, it is important also to know if they have strategies for the implementation of innovations and if the existence of strategy is associated to some factors. What we identified is that the newest companies mostly implement innovations alongside with the smallest ones. Furthermore, there are more strategic innovators among the companies, who work on world markets and use financing from national funds. And as concern the types of produced wines all of those who produce fruit wines, special wines, and wines with PDO are strategic innovators.

We should note that these results require a further research to identify the reason for identified associations.

The research is part of a project KP-06-M25/5/17.12.2018, financed by National Science Fund of Bulgaria, Ministry of Education and Science.

\section{References}

1. M. Angelova, D. Pastarmadzhieva, G. Dimitrova, P. Georgiev, Innovative Practices in Wine Industry: Opportunities for Competitiveness Enhancement in Bulgaria, Proceedings of IEEE International Conference on Creative Business for Smart and Sustainable Growth (CREBUS), 18-21 March 2019, Sandanski, Bulgaria (2019)

2. Chen Jin, Xu Qing-rui, J. Zhejiang Univ. Sci A, 1(2), 229-232 (2000)

3. Regional Innovation Strategy Sachsen-Anhalt 2014-2020, https://mw.sachsenanhalt.de/fileadmin/Bibliothek/Politik_und_Verwaltung/MW/Publikationen/RIS/Regio nal_Innovation_Strategy_Sachsen-Anhalt.pdf

4. Y. Lu, E. W. K. Sang, M. W. Peng, Asia Pacific J Manage, 25(3), 361-374 (2008)

5. W. Vanhaverbeke, N. Peeters, Creativity Innov. Manag., 14(3), 246-256 (2005)

6. C. Peeters, B. van Pottelsberghe de la Potterie, J. Evol. Econ., 16, (1-2), 109-135 (2006)

7. C. A. Enz, S. A. Way, Serv. Sci., 8(2), 97-107 (2016)

8. T. Sauber, H. Tschirky, Structured Creativity. Formulating an Innovation Strategy, (Basingstoke, UK: Palgrave Macmillan, 2006)

9. I. Stankevice, G.Jucevicius, Socialiniai mokslai. 69(3), 24-31 (2010)

10. Sh. D. Saleh, Cl. K. Wang, IEEE Trans. Eng. Manage, 40 (1), 14-21 (1993)

11. A. Belasen, E.B. Luber, Innovation Implementation: Leading from the Middle Out, In Nicole Pfeffermann and Julie Gould (Eds), Strategy and Communication for Innovation Integrative Perspectives on Innovation in the Digital Economy (3rd ed.), Cham, Switzerland: Springer International Publishing AG, 229-243 (2017)

12. J. Su Kim, G. H. Chung, IOM, 19(3), 372-399 (2017)

13. M. Angelova, D. Pastarmadzhieva and G. Dimitrova, Conceptual Model for Investigating the Innovation Activity of Enterprises, in Proceedings of V International 
scientific conference "High Technologies. Business. Society 2020", 09-12 March 2020, Bulgaria (2020)

14. Strategic Innovation Group, A Framework for Strategic Innovation from Breakthrough Inspiration to Business Impact, (Piedmont, CA. 510.496.6020), (2020), [Online], http://www.strategicinnovationgroup.com

15. M. Angelova, D. Pastarmadzhieva, Application of Innovations in SMEs from Wine Industry in Bulgaria: The Case of Plovdiv Region, in Proceedings of International conference on High Technology for Sustainable Development HiTech 2019, 8 - 9 October 2019, Bulgaria (2019) 